\title{
Uma Abordagem Ciente de Contexto para Criação e Realização de Atividades Restritas por Localização em EaD
}

\author{
Wesley de S. Viana, Elaine H. T. Oliveira \\ Instituto de Computação - Universidade Federal do Amazonas (UFAM) \\ Caixa Postal 69077 - 000 - Amazonas - AM - Brasil \\ $\{$ wesley, elaine\} @i comp.ufam.edu.br
}

\begin{abstract}
The distance education is a technology-mediated education mode in which the teacher and pupil develop their functions within an online environment. In this context after a couple of interviews with teachers of the distance course focused on stage, it has been found difficulties of some teachers to follow students in designated locations for supervised internship, both physically and by E-learning support environment. Soon, the present work proposes an approach involving context-aware mobile technologies to AVA in order to enable the preparation and realization of tasks in specific locations. The approach was validated in two disciplines of stage on which demonstrated good degrees of acceptance.
\end{abstract}

Resumo. A Educação a Distância é uma modalidade de ensino mediada por tecnologia no qual o professor e aluno desenvolvem suas funções dentro de um ambiente online. Nesse contexto após algumas entrevistas com professores de curso a distância voltado para estágio, foi constatado dificuldades de alguns professores em acompanhar os alunos em locais designados para a realização de estágio supervisionado, tanto fisicamente quanto pelo ambiente de suporte à EaD. Logo, o presente trabalho propõe uma abordagem ciente de contexto envolvendo tecnologias móveis para AVA a fim de possibilitar a elaboração e a realização de tarefas em locais específicos. A abordagem foi validada em duas disciplinas de estágio no qual demonstrou bons graus de aceitação.

\section{Introdução}

O surgimento de novas tecnologias tem provocado mudanças significativas na área da educação, pois além contribuir na produção de conhecimento também possibilita novas formas de aprender com a utilização de tecnologias, modificando as formas tradicionais de ensino e possibilitando o surgimento de diversos ambientes interativos voltados a educação. Nesse contexto, a Educação à Distância $(\mathrm{EaD})$ surge como uma modalidade de ensino que utiliza a tecnologia na mediação do conhecimento entre professor e aluno. Segundo Mugnol (2009), tal modalidade é centrada no aluno e mediada pelas tecnologias da sociedade da informação, levando à necessidade de se investigar como alunos e instrutores com o uso das novas tecnologias podem colaborar para gerar novos conhecimentos.

Nos últimos anos, os cursos de $\mathrm{EaD}$ são constantemente alvo de pesquisas, sendo o seu processo de ensino-aprendizagem considerado muito complexo. Contudo, 
existem algumas limitações como: a falta de suporte para verificar as interações sociais, acompanhar as atividades dos alunos, prever evasão de alunos entre outros. Algumas dessas limitações são evidenciadas em: Alencar e Neto (2012), Lima et al (2014), e Campana et al (2008).

Em cursos a distância o estágio curricular é considerado uma oportunidade que o aluno tem de aplicar as teorias aprendidas na prática, se preparando para sua carreira profissional [Freire et al 2014]. Tal afirmação é corroborada por Pimenta e Lima (2010) que consideram o estágio como um campo do conhecimento que não deve ser reduzido apenas a uma atividade prática instrumental e sim em uma produção na interação dos cursos de formação com o campo social no qual se desenvolvem as práticas educativas, podendo nesse sentido ser considerado como uma atividade de pesquisa.

Após algumas entrevistas com professores de duas instituições de ensino, foi possível constatar a dificuldade do professor em acompanhar as visitas dos alunos aos locais designados para estágio em cursos à distância. Essas dificuldades estão relacionadas a acompanhar se os alunos estão frequentando o local de estágio, e ao mesmo tempo utilizar o ambiente AVA para designar essa função.

A estratégia de solução para o proposto trabalho busca investigar em turmas de estágios a utilização de tecnologias móveis por meio de uma abordagem ciente de contexto por meio do aplicativo móvel e um modulo para criar atividades restritas por localização em um AVA, de modo a possibilitar criar atividades e restringir por localizações geográficas especificas para certificar que o aluno esteve presente no local estabelecido pelo professor.

\section{Fundamentação Teórica}

Esta Seção descreve a fundamentação teórica utilizada para o embasamento do trabalho.

\subsection{Mobile Learning e Computação Ciente de Contexto}

A presença das tecnologias móveis vem se tornando cada vez mais presente no cotidiano das pessoas, promovendo novas formas de acesso à informação, ensino e aprendizado, dinamizando a busca de informações e possibilitando o uso da sua maior vantagem, que é a mobilidade [Oliveira et al 2014]. Neste sentido, estamos vivendo em uma sociedade cada vez mais conectada, com uma variedade de fontes de informação e modos de comunicação disponíveis em locais de trabalho, residências, instituição de ensino e em outros espaços.

Neste contexto Mobile Learning pode ser definido como uma área de estudo que permite que a troca de informação passe de um ambiente físico para outros espaços com a utilização de dispositivos móveis. Este novo conceito está presente na área educacional, permitindo aos alunos estabelecer autonomia na aprendizagem e fornecer aos instrutores e administradores de educação um ensino flexível. Além disso, a aprendizagem móvel pode ser considerada o futuro da Educação a Distância [Jin 2009].

O conceito de Ciência de Contexto (Context-Aware) pode ser definido como a capacidade de uma aplicação móvel reconhecer e reagir a mudanças no ambiente em que o usuário está situado [Schilit e Theimer 1994]. Seguindo a mesma linha de 
raciocínio, para Satyanarayanan (2001), para um sistema ser ciente de contexto, ele deve ser consciente do estado e do seu usuário, e deve modificar seu comportamento com base nessas informações. Dessa forma, é possível concluir que essa área de estudo possui alguns dos principais alvos de pesquisa e utilização, centrada no usuário e no ambiente que ele interage.

Nesse trabalho, foi utilizado o contexto por localização, abordado em alguns trabalhos na literatura como Location Aware, podendo ser definida como serviços sensíveis ao contexto que utilizam a localização do usuário para adaptar um serviço de acordo com o seu interesse [Kaasinen 2003]. Trata-se de uma área que está preocupada com informações de contexto por localização, seja ela por GPS, localização indoor ou por triangulação.

\section{Trabalhos Correlatos}

Alguns trabalhos abordam a utilização do conceito de Mobile Learning com eventos locativos para possibilitar experiências de aprendizagem. No projeto Alra [Santos et al 2013], temos um jogo locativo de realidade aumentada, onde os participantes interagem com objetos do ambiente dispostos em um mapa simulado no dispositivo móvel para cumprir missões e conquistar resultados. Este projeto propõe aos professores e alunos a possibilidade de conceber e vivenciar novas experiências de aprendizagem. No trabalho de Rodrigues et al (2010) propõe um aplicativo ciente de contexto por localização com o LMS (Learning Management System) Aulanet, onde os estudantes realizam tarefas e são avaliados em posições geográficas pré-definidas e ao termino das atividades os resultados são sincronizados com um repositório com o LMS Aulanet.

No trabalho de Chu et al (2010), temos um sistema de aprendizagem móvel que combina recursos do mundo real com o mundo digital através do uso de tecnologia de identificação por radiofrequência (RFID). Essa combinação detectar e analisa comportamentos reais de aprendizagem dos alunos. O sistema proposto foi utilizado em uma turma de ciências naturais que, com a ajuda da tecnologia de sensoriamento, o sistema de aprendizagem móvel detecta a localização de cada estudante e orienta na localização das plantas alvo ao chegar ao local o sistema mostrar informações sobre a planta-alvo.

O trabalho de Li et al (2012), descreve uma arquitetura centralizada com a integração de dispositivos móveis. $\mathrm{O}$ usuário fotografa um objeto de seu interesse e o mesmo é enviado a um servidor. Após o servidor identificar o local onde a imagem foto fotografada, ele retorna o conteúdo textual associado com o objeto de interesse do aluno.

\section{Abordagem do Trabalho}

A abordagem do trabalho proposto é constituída pelo aplicativo MobiLMS customizado, gerado para Android 4.0 ou superior, um plugin de formato de curso baseado no plugin "Kamedia GPS" [Kamedia 2013] voltado ao Moodle baseado no plugin para permitir criar e disponibilizar atividades restritas por localização no ambiente Moodle e um plugin para execução de webservices de modo a possibilitar executar as funcionalidades da abordagem no aplicativo MobiLMS. 
Na Figura 1 temos a visão geral da abordagem, onde estabelece as principais funcionalidades realizadas pelo professor (Moodle desktop) e aluno (Aplicativo MobiLMS).

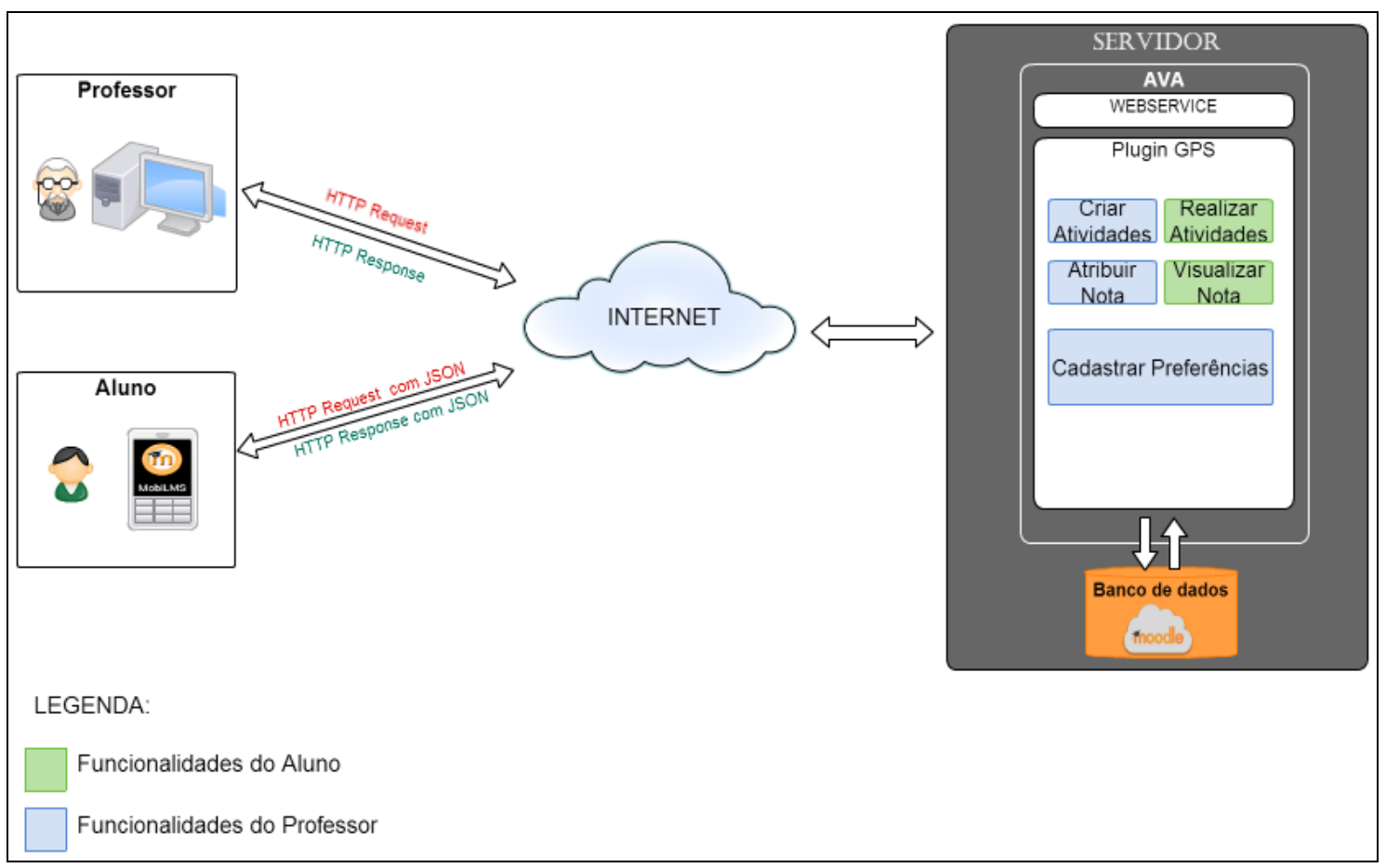

Figura 1. Visão Geral da Abordagem.

A Figura 1 mostra a visão geral da abordagem, mostrando que o professor utiliza o ambiente AVA pelo computador para executar as funcionalidades da abordagem, enquanto que os alunos executam as funcionalidades pelo celular por meio do aplicativo MobiLMs.

A atividade é criada no ambiente Moodle por meio de utilização da funcionalidade "adicionar uma atividade ou recurso" e "adicionar uma tarefa". Em seguida deve-se restringir por localização através do acesso ao sumário de um tópico. $\mathrm{O}$ aluno deve realizar a atividade utilizando o aplicativo MobiLMS no qual responde a tarefa por meio de um texto com a possibilidade de tirar uma foto pela câmera do dispositivo e anexar na tarefa. Feito o procedimento a atividade é submetida ao ambiente e o professor deve corrigir a atividade.

A execução do MobiLMS se estabelece pela conexão no AVA Moodle por meio de webservices. O Json (JavaScript Object Notation) foi utilizado para captar a respostas de cada função estendida. A interação do aplicativo com o Google Maps API se estabelece através de um plugin disponível para o Phonegap. A Figura 2 ilustra a criação da tarefa em que o professor cria uma tarefa no AVA Moodle e restringe por localização em um mapa. 


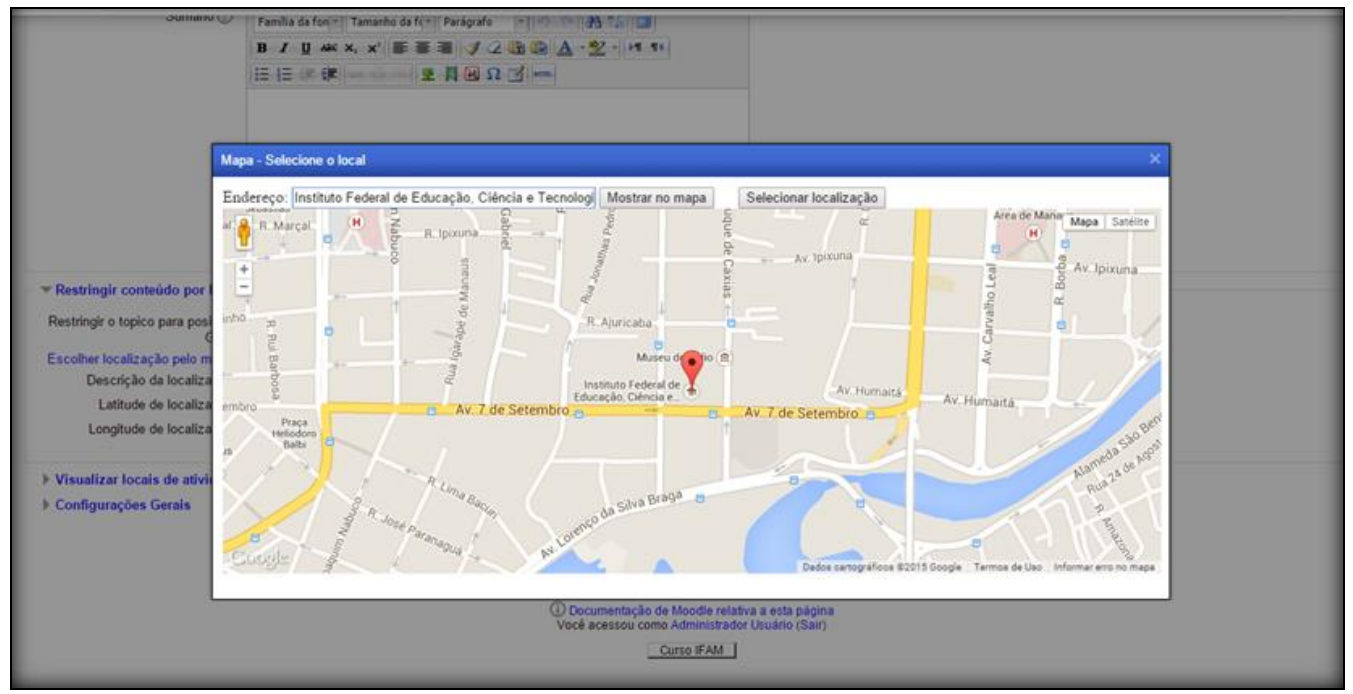

Figura 2. Criando Atividades Restritas pelo Mapa.

Nas Figuras 3 e 4, é possível visualizar as telas do aplicativo. A Figura 3 aborda a tela inicial de um curso estruturado em tópicos e a Figura 4 mostra o aplicativo reconhecendo a localização do aluno e mostrando as localizações de atividades no mapa. Na Figura 3, o tópico pintado de verde indica um conteúdo disponível na localização geográfica atual do aluno e o tópico na cor cinza indica um tópico bloqueado, no qual o acesso ao conteúdo é possível em outra posição geográfica.

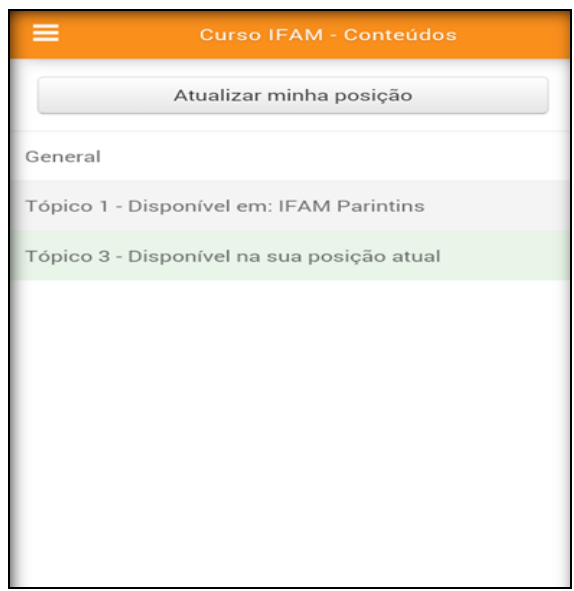

Figura 3. Tela do Curso.

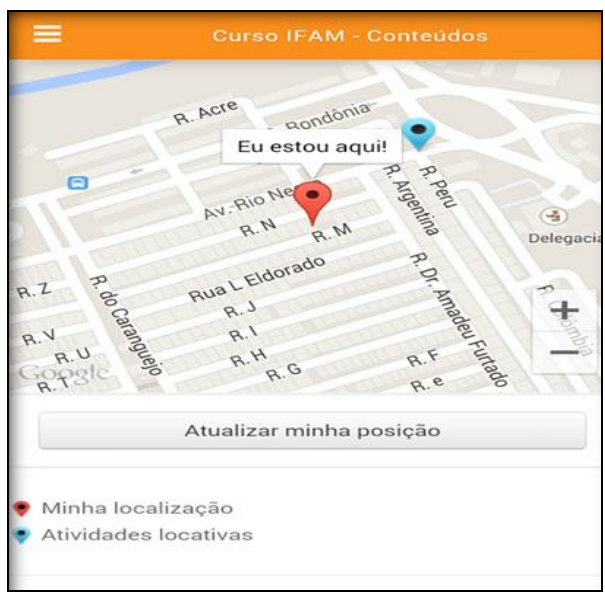

Figura 4. Localizações no Mapa.

\section{Avaliação e Descrição dos Cenários de Aplicação}

Foram realizados experimentos em duas turmas reais de estágios: no curso de Agente Comunitário de Saúde pelo IFAM (Instituto Federal do Amazonas) localizado em Parintins - AM e no curso de Tecnólogo em Análise e Desenvovimento de Sistemas pelo IFRR (Instituto Federal de Roraima), localizado em Boa Vista - RR com um professor em cada instituição e seis alunos no total.

A validação da abordagem constitui de avaliação buscando investigar o grau de aceitação da abordagem em cursos reais. Dessa forma foi buscado explorar a aceitação da utilidade da abordagem por meio da avaliação em turmas de estágios. O método de 
avaliação foi por meio aplicação de questionários aos alunos e professores envolvidos, que segundo Laguardia et al [2007] trata-se do método talvez mais utilizado nos diversos tipos de avaliação de cursos online.

Os questionários são constituídos de perguntas onde o professor e alunos deveriam marcar como resposta uma das seguintes alternativas: "Concordo plenamente", "Concordo parcialmente", "Concordo em pequena parte", e "Discordo", e no fim continha um espaço para acrescentar informações que considere importante sobre as perguntas dos questionários.

\section{Resultados e Discussão}

Os questionários aplicados constituem de: um questionário ao professor voltado para avaliar a utilidade do módulo de criação de tarefas no Moodle para serem disponibilizadas em posições geográficas definidas e, um aos alunos em que buscou-se avaliar a utilidade do aplicativo MobiLMS no decorrer do estágio. A Tabela 1 mostra os as informações sobre utilidade obtidas dos professores.

Tabela 1. Dados sobre Utilidade do Módulo para o Professor.

\begin{tabular}{|l|c|c|c|c|}
\hline Perguntas respondidas & $\begin{array}{c}\text { Concordou } \\
\text { plenamente }\end{array}$ & $\begin{array}{c}\text { Concordou } \\
\text { em grande } \\
\text { parte }\end{array}$ & $\begin{array}{c}\text { Concordou } \\
\text { em pequena } \\
\text { parte }\end{array}$ & Discordou \\
\hline $\begin{array}{l}\text { Possibilitou criar várias } \\
\text { tarefas durante o estágio. }\end{array}$ & 2 & 0 & 0 & 0 \\
\hline $\begin{array}{l}\text { Permitiu imaginar/criar } \\
\text { vários tipos de tarefas, }\end{array}$ & 2 & 0 & 0 & 0 \\
\hline $\begin{array}{l}\text { Contribuiu em certificar } \\
\text { da visita dos alunos aos } \\
\text { locais de estágio. }\end{array}$ & 1 & 1 & 0 & 0 \\
\hline $\begin{array}{l}\text { Eu considero o módulo } \\
\text { útil para utilizar no } \\
\text { estágio. }\end{array}$ & 2 & 0 & 0 & 0 \\
\hline
\end{tabular}

De acordo com as informações da Tabela 1 foi possível concluir que o módulo apresentou bons resultados sobre a utilidade do modulo de criação de tarefas restritas por localização no estágio, contudo a informação sobre considerar que o módulo possibilitou o acompanhamento dos alunos nos locais de estágios teve opiniões diferentes, permitindo concluir que esse aspecto deve ser melhorado para assim possibilitar um melhor acompanhamento dos alunos nos locais de estágio. Na Tabela 2 é possível visualizar os dados obtidos dos alunos ao utilizarem o aplicativos MobiLMS para realizar as atividades. 
Tabela 2. Dados da Utilidade do Aplicativo.

\begin{tabular}{|l|c|c|c|c|}
\hline \multicolumn{1}{|c|}{ Perguntas respondidas } & $\begin{array}{c}\text { Concordou } \\
\text { plenamente }\end{array}$ & $\begin{array}{c}\text { Concordou } \\
\text { em grande } \\
\text { parte }\end{array}$ & $\begin{array}{c}\text { Concordou } \\
\text { em pequena } \\
\text { parte }\end{array}$ & Discordou \\
\hline $\begin{array}{l}\text { O aplicativo auxiliou a realizar } \\
\text { as tarefas de forma rápida. }\end{array}$ & 6 & 0 & 0 & 0 \\
\hline $\begin{array}{l}\text { Utilizar o aplicativo ajudou na } \\
\text { utilização do ambiente Moodle. }\end{array}$ & 5 & 1 & 0 & 0 \\
\hline $\begin{array}{l}\text { Usar o aplicativo facilitou a } \\
\text { realização das tarefas. }\end{array}$ & 6 & 0 & 0 & 0 \\
\hline $\begin{array}{l}\text { O aplicativo me deixou } \\
\text { motivado durante o estágio. }\end{array}$ & 5 & 1 & 0 & 0 \\
\hline $\begin{array}{l}\text { Eu considero o aplicativo útil } \\
\text { para utilizar no decorrer do } \\
\text { estágio. }\end{array}$ & 6 & 0 & 0 & 0 \\
\hline
\end{tabular}

Com os dados da Tabela 2, pode-se concluir que: nos quesitos do aplicativo auxiliar a realizar as tarefas de forma rápida e considerar o aplicativo útil para se utilizar em estágios todos os alunos concordaram plenamente. Nos outros dois quesitos relacionados sobre deixar motivado e ajudar na utilização do Moodle as opiniões foram divididas.

De acordo com informações coletadas nos espaços para comentários das questões constituintes dos questionários foi possível constatar algumas limitações da abordagem, tais limitações foram relacionadas à imprecisão do GPS para estabelecer a localização em algumas localizações geográficas, lentidão da aplicação na execução em internet lentas como a $2 \mathrm{G}$ e lentidão para anexar fotos à tarefa.

\section{Considerações Finais}

No presente trabalho é constituído de uma abordagem ciente de contexto envolvendo tecnologias móveis e um módulo para o professor no Moodle convencional. Isso possibilita ao professor a criação de atividades restritas por localização em AVAs e, ao mesmo tempo, permite aos alunos realizarem as atividades por meio do aplicativo.

Existem várias outras possibilidades de aplicação da abordagem descrita nesse trabalho, como por exemplo, o desenvolvimento de atividades práticas de campo, no qual o professor prepara uma atividade de campo em um local específico, exigindo aos alunos o deslocamento para o local a fim de realizar a tarefa, tornando o aprendizado mais dinâmico e conveniente. Várias outras atividades são possíveis para aplicações tais como: disponibilizar conteúdos informativos para passeios em zoológicos e museus, reuniões e aulas em locais definidos, visitas técnicas, passeio turísticos, entre outros.

De acordo com as informações coletadas dos estudantes e professores, pode-se considerar a abordagem útil para utilizar em disciplinas de estágio, contudo o fato de não concordarem em alguns pontos indicam que existem pontos a serem melhorados na abordagem.

Algumas limitações constatadas apontam que a abordagem deve ser implementada para não depender de internet, pois em algumas regiões existem 
problemas com o sinal de internet. Outro ponto que merece destaque foi relacionado a lentidão de upload de fotos em que foi necessário utilizar na abordagem um algoritmo para compressão de imagem, diminuindo o tamanho da imagem e consequentemente melhorando consideravelmente o tempo de upload.

\section{Agradecimentos}

Os autores agradecem o apoio financeiro da Fundação de Amparo à Pesquisa do Estado do Amazonas (FAPEAM) através do Edital No 016/2013, Projeto PROTIPesquisa sob o processo $\mathrm{N}^{\circ}$ 062.00597/2014. Além disso, parte dos resultados apresentados neste trabalho foi obtida através de atividades de P\&D do "Programa de Qualificação em Grande Escala em Tecnologias Móveis - PROMOBILE", projeto patrocinado pela Samsung Eletrônica da Amazônia Ltda. nos termos da lei federal brasileira $\mathrm{n}^{\circ} 8.248 / 91$.

\section{Referências}

Alencar, M. A. D. S., Netto, J. F. M. (2012) "Sistema Multiagente para Apoiar a Percepção e o Acompanhamento de Atividades em Ambientes Virtuais de Aprendizagem“. Anais do Simpósio Brasileiro de Informática na Educação - SBIE. Vol. 23, Rio de Janeiro.

Campana, V. (2008) "Agentes para Apoiar o Acompanhamento das Atividades em Ambientes Virtuais de Aprendizagem”. Anais do Simpósio brasileiro de Informática na Educação, p. 62-73, Fortaleza.

Chu, Hwang, Tsai and Tseng. (2010)" A two-tier test approach to developing locationaware mobile learning system for natural science course". Computers \& Education, p. 1618-1627, Reino Unido.

Freire, W. C., Lima, B. P. D. D. C. (2014) "Estágio Curricular na EaD: Desafios e Possibilidades na Licenciatura de um Polo UAB”. REVELLI - Revista de Educação, Linguagem e Literatura, Vol. 5, p. 81-99, Goiás.

Kaasinen, E. (2003) "User Needs for Mocation-Aware Mobile Services. Personal and Ubiquitous Computing" Vol 7, p 70-79, ACM.

Kamedia GPS Format free. URL: <https://moodle.org/plugins/view/format_gps>. Acessado em Agosto de 2013.

Laguardia, J., Portela, M. C., Vasconcellos M. M. (2007) "Avaliação em Ambientes Virtuais de Aprendizagem”. Educação e Pesquisa, Vol 33, p. 513-530,

Jin, Y. (2009) "Research of one Mobile Learning System" International Conference on Wireless Networks and Information Systems - WNIS. IEEE, p. 162-165, China.

Li, Y., Guo, A., Lee, J. A., Gao, Y., e Ling, Y. L. (2012) "Visual Interactive and Location Activated Mobile Learning". IEEE Seventh International Conference on Wireless, Mobile and Ubiquitous Technology in Education, Japão.

Lima, D. P. R., Netto, J. F., Gaspar, W. (2014) "Um Sistema Multiagente que Caracteriza as Relações Sociais entre Alunos de um Ambiente Virtual de 
Aprendizagem" Anais do Simpósio Brasileiro de Informática na Educação - SBIE, Vol. 25, p.1163-1172, Dourados.

Lima, M. S. L.; Pimenta, S. G (2010). "Estágio e Docência: Diferentes Concepções”. Poíesis Pedagógica, Vol. 3, p. 5-24, Goiás.

Mugnol, M. (2009) "A Educação a Distância no Brasil: Conceitos e Fundamentos" Revista Diálogo Educ. Vol. 9, p. 335-349, Curitiba.

Oliveira, J. B., de Queiroz, F. N., Hypólito, V. A. H. A., Hypólito, J. M. (2014) “As Tecnologias Móveis como Contribuintes no Processo de Ensino e Aprendizagem na EAD”. Simpósio Internacional de Educação a Distância e Encontro de Pesquisadores em Educação a Distância - SIED: EnPED, São Paulo.

Rodrigues, J. J., Veiga, I. D., e Vaidya, B. (2010) "Implementation of Location-Aware M-Learning System". Second International Conference on Mobile, Hybrid, and Online Learning. IEEE, p. 82-86, Portugal.

Santos, O. L. Rafalski, J. Menezes, C. S. (2013) "Uma Game Engine para Aventuras Pedagógicas Locativas em Realidade Aumentada". Anais do Simpósio Brasileiro de Informática na Educação - SBIE. Vol. 24, São Paulo.

Satyanarayanan, M. (2001) "Pervasive Computing: Vision and Challenges". Personal Communications, IEEE, Vol. 8, p. 10-17.

Schilit, B., Theimer, M. (1994) "Disseminating Active Map Information to Mobile Hosts”. IEEE Network, p. 22-32. 